\title{
Caspase-11 Mediates Pyroptosis of Tubular Epithelial Cells and Septic Acute Kidney Injury
}

\author{
Zhiming Yea Li Zhang $^{a}$ Ruizhao Lia Wei Donga Shuangxin Liu ${ }^{a}$ \\ Zhilian Li $^{a}$ Huaban Liang ${ }^{a}$ Lifen Wang $^{a}$ Wei Shi $^{a}$ Asrar B. Malik ${ }^{b}$ \\ Kwong Tai Cheng ${ }^{b}$ Xinling Liang ${ }^{a}$ \\ aDivision of Nephrology, Guangdong Provincial People's Hospital, Guangdong Academy \\ of Medical Sciences, Guangzhou, China; ${ }^{b}$ Department of Pharmacology and Division of \\ Cardiology, Department of Medicine, The University of Illinois College of Medicine, \\ Chicago, IL, USA
}

\section{Keywords}

Caspase-11 · Pyroptosis · Tubular epithelial cells · Sepsis · Acute kidney Injury

\begin{abstract}
Background/Aims: Acute kidney injury (AKI) is a serious complication of sepsis and has a high morbidity and mortality rate. Caspase- 11 induces pyroptosis, a form of programmed cell death that plays a critical role in endotoxic shock, but its role in tubular epithelial cell death and whether it contributes to sepsis-associated AKI remains unknown. Methods: The caspase-11/mouse received an intraperitoneal injection of lipopolysaccharide (LPS, $40 \mathrm{mg} / \mathrm{kg}$ body weight).

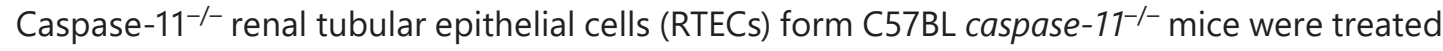
with LPS in vitro. The IL-1 $\beta$ ELISA kit and Scr assay kit were used to measure the level of interleukin-1 $\beta$ and serum creatinine. Annexin V-FITC assay and TUNEL staining assay were used to detect the cell death in different groups in vitro and in vivo. Western blot was performed to analyze the protein expression of caspase-11 and Gsdmdc1. Results: LPS-induced sepsis results in lytic death of RTECs, accompanied by increased expression of the pyroptosis-related proteins caspase-11 and Gsdmd. However, the increase in pyroptosis-related protein expression induced by LPS was attenuated with caspase- 11 knockout, both in vitro and in vivo. Furthermore, when challenged with lethal doses of systemic LPS, pathologic abnormalities in renal structure, increased serum and kidney interleukin- $1 \beta$, increased serum creatinine, and
\end{abstract}

Zhiming Ye and Li Zhang contributed equally to this study. 


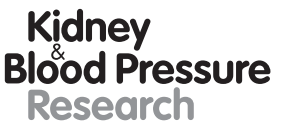

Kidney
Blood Pressure
Research

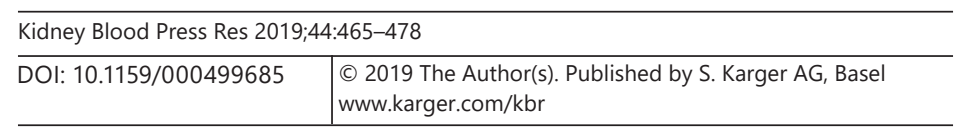

Ye et al.: Caspase-11 and Septic Acute Kidney Injury

animal death were observed in wild-type mice but prevented in caspase-11-/- mice. Conclusions: Caspase-11-induced pyroptosis of RTECs is a key event during septic AKI, and targeting of caspase-11 in RTECs may serve as a novel therapeutic target in septic AKI.

(C) 2019 The Author(s)

Published by S. Karger AG, Basel

\section{Introduction}

Acute kidney injury (AKI) is a serious complication of systemic inflammatory response syndrome, which has a high mortality rate [1-3]. Thirty to fifty percent of septic patients develop renal failure, and AKI can double the mortality rate up to $70 \%$. Limited understanding of the cellular mechanisms of sepsis-associated AKI complicates the development of an effective treatment. Sepsis-associated AKI presents with severe impairment of kidney function evident by extreme elevations in blood urea nitrogen and serum creatinine (Scr); however, only modest tubular injury is observed with minimal true necrosis. The lack of correlation between classic cell necrosis and impaired kidney function implicates alternative forms of cell death as contributors to kidney injury. Therefore, in recent years, investigators have increasingly recognized that apoptosis, necroptosis, and autophagic cell death must be considered in renal cell death [4-6].

Recently, a new form of programmed cell death - pyroptosis - has been described. Pyroptosis is morphologically and mechanistically distinct from other forms of cell death and is characterized by rapid plasma membrane rupture and release of pro-inflammatory intracellular contents [7-9]. Pyroptosis and apoptosis are both programmed mechanisms of cell death, but each process depends on different caspases, unlike oncosis. Similar to oncosis, but unlike apoptosis, pyroptosis results in cellular lysis and release of pro-inflammatory cytokines, including interleukin (IL)-1 $\beta$ and IL-18, into the extracellular space $[10,11]$. IL-1 $\beta$ is a key inflammatory cytokine involved in host defense mechanisms against pathogens and serves as a "gatekeeper" of inflammation $[9,12]$. Pyroptosis is observed in monocytes, macrophages, dendritic cells, and endothelial cells when associated with infection [12-14]. However, whether pyroptosis is involved in the death of renal tubular epithelial cells (RTECs) after exposure to microbial infection is not known.

Caspase-11 is an intracellular cysteine protease that mediates the host cell response to gram-negative bacterial pathogens and sepsis. Host cells sense lipopolysaccharide (LPS) from gram-negative bacteria via a non-canonical inflammasome pathway that ultimately results in caspase-11 activation and cell death [11,15-18]. A series of studies indicate that caspase-11 plays a critical role in pyroptosis $[11,15,19-21]$, but its potential role in renal epithelial cell pyroptosis and septic AKI remains unknown.

In the present study, we study whether caspase-11 mediates pyroptosis of tubular epithelial cells and septic AKI. We show that the endotoxin LPS induces pyroptosis of RTECs. We also demonstrate that genetic deletion of caspase-11 attenuates AKI and markedly increases cell survival, even in the presence of lethal systemic doses of LPS.

\section{Material and Methods}

Chemicals and Reagents

LPS from E. coli 0111:B4 was obtained from Sigma-Aldrich (L2630, Sigma, St. Louis, MO, USA). Mouse IL-1 $\beta$ ELISA kit was purchased from R\&D Systems, Inc. (Lot 324909, Minneapolis, MN, USA). Antibodies against caspase-11 and Gsdmdc1 were obtained from 
Kidney
Blood Pressure

Research

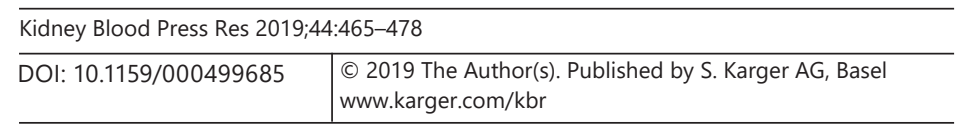

Ye et al.: Caspase-11 and Septic Acute Kidney Injury

Sigma-Aldrich (c1354, Sigma) and Santa Cruz Biotechnology (sc-393656, Santa Cruz, CA, USA), respectively. SCr assay kit reagents were supplied by Abcam (ab65340, Cambridge, MA, USA).

\section{Animals}

Mice were bred and maintained under specific pathogen-free conditions at the University

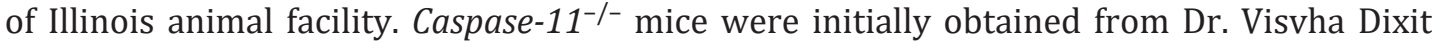
(Genentech). The caspase-11/- mouse colony was maintained at a pathogen-free animal housing facility at the University of Illinois at Chicago (UIC). C57BLk/6J mice initially acquired from The Jackson Laboratory and bred at UIC were used as wild-type (WT) controls. Male mice, aged 8-12 weeks old, were used for all experiments.

\section{Endotoxin-Induced Acute Kidney Injury}

Mice received an i.p. injection of LPS ( $40 \mathrm{mg} / \mathrm{kg}$ body weight) or an equal volume of $0.9 \%$ saline as a control. Blood was collected for Scr and IL-1 $\beta$ measurement at times 0,6 , and $12 \mathrm{~h}$ after LPS injection, and kidneys were harvested for pathological and immunoblotting analyses.

\section{Cell Culture}

The caspase-11-/- RTEC cell line was supplied by Cell Biologics Inc. (Chicago, IL, USA).

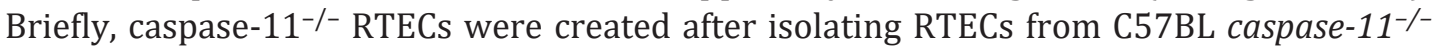
mice. Renal cortex was isolated, minced with a sterile blade, and then filtered through a 40 $\mu \mathrm{m}$ filter before being pelleted and resuspended in defined media. Cells were trypsinized and characterized by FACS analysis using anti-CD326 antibodies, which are known epithelial cell surface markers. RTECs were grown in a 50:50 mixture of DMEM/Ham's F-12 supplemented with 5\% fetal bovine serum (Life Technologies Corporation, Grand island, NY, USA).

\section{Annexin V-FITC Assay}

Cell death in different groups was determined using an Annexin V-FITC apoptosis detection kit, according to the manufacturer's protocol (K101-400, BioVision Incorporated, Milpitas, CA, USA). Briefly, the cell pellet was resuspended in $1 \times$ binding buffer, followed by incubation with $5 \mu \mathrm{L}$ of Annexin V (conjugated with FITC) and $5 \mu \mathrm{L}$ of PI in the dark for 10 min. Cell fluorescence was then analyzed with a Cell Lab QuantaTM SC flow cytometer (Beckman Coulter, Inc., Fullerton, CA, USA). Cells positive for Annexin V-FITC and negative for PI were considered to be undergoing cell death. All experiments were repeated three times.

\section{TUNEL Assay}

The TUNEL staining assay was conducted using the in situ Cell Death Detection kit from Roche Applied Science (Indianapolis, IN, USA), according to the manufacturer's instructions. Briefly, paraffin-embedded tissue sections were deparaffinized using a standard protocol and permeabilized with $0.1 \mathrm{M}$ sodium citrate $\left(\mathrm{pH} 6.0\right.$ ) at $65^{\circ} \mathrm{C}$ for $1 \mathrm{~h}$. Tissue sections were incubated with the TUNEL reaction buffer for $1 \mathrm{~h}$ at $37^{\circ} \mathrm{C}$ in a humidified chamber.

Positive staining was detected by confocal microscopy (LeicaSP5-FCS, Wetzlar, Germany). For each section, 10-20 fields were randomly selected and positively stained cells were counted.

\section{Histological Analysis and Tubular Injury Score}

For histological analysis, kidney tissues were fixed with 4\% paraformaldehyde overnight and embedded in paraffin. The paraffin sections $(4 \mu \mathrm{m})$ were stained with H\&E. The quantitative analysis of the renal tubular injury used the grading scores proposed in a previous 


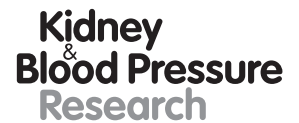

Kidney
Blood Pressure
Research

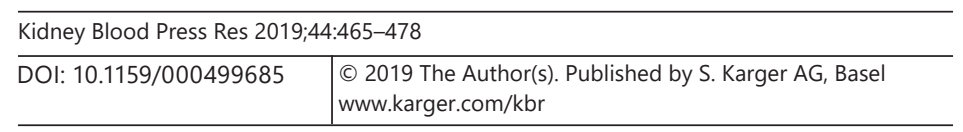

Ye et al.: Caspase-11 and Septic Acute Kidney Injury

study [22]. The sections were evaluated by assessing 10 randomly selected high-power fields (40× objective), with higher scores representing more severe damage, as follows: tubular epithelial cell flattening ( 1 point), brush border loss ( 1 point), cell membrane bleb formation (1 or 2 points), interstitial edema (1 point), cytoplasmic vacuolization (1 point), cell necrosis (1 or 2 points), and tubular lumen obstruction (1 or 2 points).

\section{Biochemical Measurements}

Blood samples were collected from the retro-orbital venous plexus and centrifuged in glass tubes at $4{ }^{\circ} \mathrm{C}$ for $10 \mathrm{~min}$ at $1,400 \mathrm{~g}$; the serum was stored at $-80^{\circ} \mathrm{C}$ in polystyrene tubes until use. The concentration of Scr was analyzed using commercial kits at the end of the experiment. The absorbance was measured using a SpectraMax M2 Multi-Mode microplate reader (Molecular Devices, Sunnyvale, CA, USA).

\section{ELISA Analysis of IL-1ß in Serum and Kidney Tissue}

Serum and kidney levels of IL-1 $\beta$ were measured with an ELISA kit, following the manufacturer's instructions (Quantikine, R\&D Systems, Inc., Minneapolis, MN, USA). Briefly, each kidney tissue was homogenized in phosphate-buffered saline (PBS) at a 1:10 concentration ( $1 \mathrm{~g}$ of tissue to $10 \mathrm{~mL}$ of PBS buffer). A monoclonal antibody specific for each protein was pre-coated onto a microplate. The standards and test samples were then pipetted into the wells to allow binding of these proteins to the immobilized antibodies. After washing away any unbound substances, an enzyme-linked polyclonal antibody specific for each protein was added to the wells. Following removal of unbound antibody-enzyme reagent through washing, a substrate solution was added to the wells and color was developed. The optical density of each well was measured at a wave length of 450 or $510 \mathrm{~nm}$ on a microreader (S190, Molecular Devices). The concentration of each cytokine or chemokine was accordingly calculated.

\section{Protein Extraction and Western Blot Analysis}

Cells treated with LPS $(30 \mu \mathrm{g} / \mathrm{mL})$ were lysed and homogenized in lysis buffer immediately. The kidney tissue samples were homogenized on ice, followed by centrifugation at $12,000 \mathrm{~g}$ for $30 \mathrm{~s}$. The proteins were extracted using a total protein extraction kit (Millipore, Billerica, MA, USA), according to the manufacturer's instructions. Protein concentrations were determined with a BCA protein assay kit (Pierce, Rockford, IL, USA). Protein samples were separated by SDS-PAGE and transferred to a PVDF membrane. The membrane was blocked with Tris buffered saline containing 5\% non-fat dry milk at room temperature for $2 \mathrm{~h}$. For immunoblotting, the membranes were incubated overnight at $4{ }^{\circ} \mathrm{C}$ with antibodies directed against caspase- 11 or Gsdmd, and $\beta$-actin $(1: 1,000)$ was used as a loading control. The secondary antibody (horseradish peroxidase conjugated anti-rabbit IgG antibody) was incubated at room temperature for $2 \mathrm{~h}$. Protein was detected using Super Signal ${ }^{\mathbb{R}}$ West Pico Chemiluminescent Substrate (Pierce Biotechnology, Rockford, IL, USA). Membranes were scanned and quantified with NIH ImageJ software. The expression of the protein examined was normalized to $\beta$-actin expression. Results were expressed as fold increase over control.

\section{Statistical Analysis}

Statistical analysis was performed using the statistical package SPSS for Windows ver. 19.0 (SPSS, Inc., Chicago, IL, USA). Data were analyzed with two-tailed unpaired Student's $t$ test for comparisons of two groups or one-way ANOVA of the repeated experiments followed by the Tukey's post hoc pairwise multiple comparisons. A $p$ value of $<0.05$ was considered significant. For all bar graphs, the mean \pm SEM is plotted. All in vitro experiments were repeated at least three times unless otherwise indicated. 


\section{Kidney \\ Blood Pressure \\ Research}

\begin{tabular}{l|l}
\hline Kidney Blood Press Res 2019;44:465-478 \\
\hline DOI: 10.1159/000499685 & $\begin{array}{l}\text { @ 2019 The Author(s). Published by S. Karger AG, Basel } \\
\text { www.karger.com/kbr }\end{array}$ \\
\hline
\end{tabular}

Ye et al.: Caspase-11 and Septic Acute Kidney Injury

\section{Results}

\section{Endotoxic Acute Kidney Injury Is Mediated by Caspase-11}

To study whether caspase-11, a known pyroptosis regulatory enzyme, was involved in sepsis-associated AKI, we assessed caspase-11 expression in caspase-11-/- RTECs treated with LPS. Isolated RTECs from WT mice or caspase-11/-- mice were cultured in vitro and treated with LPS $(30 \mu \mathrm{g} / \mathrm{mL})$ for 6,12 , or $24 \mathrm{~h}$. As shown in Figure 1a, b, immunoblotting revealed that protein expression of caspase- 11 was markedly increased in LPS-treated RTECs from WT mice after $6 \mathrm{~h}$, and a stronger expression was observed after $12 \mathrm{~h}$. However, this effect was blocked in LPS-treated caspase-11/-/ RTECs. Similar results also demonstrated that caspase-11 protein expression was significantly increased in renal tissues isolated from WT mice treated with LPS (40 mg/kg), and this effect was also inhibited in LPS-treated caspase$11^{-/-}$mice (Fig. 1c, d).

To evaluate histopathological characteristics of kidneys, renal tissue sections were H\&Estained (Fig. 1e). Histopathological examination revealed LPS-induced kidney damage in WT mice, characterized by renal tubular degeneration and dilatation with variable flattening of the cytoplasm, and loss of the brush border. In contrast, the extent of observed LPS-induced kidney injury was reduced in caspase-11 knockout mice (Fig. 1e). Quantitative analysis further revealed that renal tubule damage scores were significantly improved in caspase-11 knockout mice compared to WT mice following LPS treatment (Fig. 1f). In addition, caspase-11 knockout mice treated with LPS also showed significantly lower levels of Scr compared with LPS-treated WT mice (Fig. 1g).

\section{Knockout of Caspase-11 Alleviates Endotoxin-Induced Pyroptosis in Renal Tubular Epithelial Cells}

To further evaluate whether caspase-11 also mediates endotoxin-induced pyroptosis in

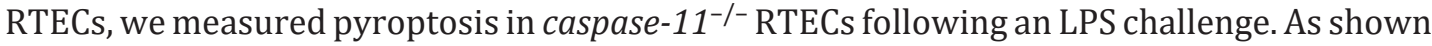
in Figure 2, there was a marked increase in pyroptosis in WT RTECs after $6 \mathrm{~h}$ of LPS treatment, and pyroptosis was further increased at $24 \mathrm{~h}$. However, in caspase-11-/- RTECs, the percent of cells undergoing pyroptosis remained constant after 0-12 h LPS treatment. Similar results were observed in mice, with WT mice displaying increased TUNEL-positive cells compared

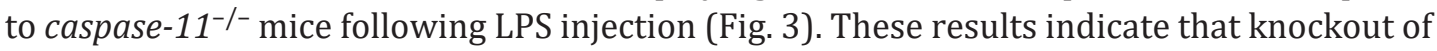
caspase-11 alleviates endotoxin-induced pyroptosis in RTECs.

Another critical target of caspase-11 is Gsdmd, which is cleaved to its active form by caspase- 11 and functions to form pores that promote cell swelling and lytic cell death $[15,19$, 23-25]. In vitro, LPS markedly increased the formation of active, cleaved Gsdmd p30 protein in renal tissues generated from WT mice (Fig. 4a). However, Gsdmd cleavage was suppressed

Fig. 1. Endotoxic AKI is mediated by caspase-11. a Isolated RTECs from wild-type (WT) mice and caspase- $11^{-/-}$mice were each cultured in vitro and treated with LPS $(30 \mu \mathrm{g} / \mathrm{mL})$ for 6,12 , or $24 \mathrm{~h}$. The protein level of caspase-11 was analyzed by western blot. b Densitometric analysis of three repetitions of caspase-11 protein in RTECs shown in b. c The protein level of caspase-11 was analyzed by western blot in isolated renal

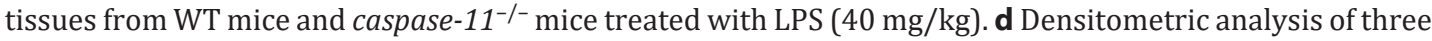
repetitions of caspase-11 protein in renal tissues shown in $\mathbf{d}$. e Pathological changes in the kidney after LPS challenge, as shown by H\&E staining. Luminal debris, loss of brush borders, and lumen expansion are observed in the injured groups. $\mathbf{f}$ Palle scores for renal tubular injury. $\mathbf{g}$ Serum creatinine was determined 6 and $12 \mathrm{~h}$ after LPS challenge ( $n=4$ animals/group). Data are shown as mean \pm SEM for each group. Different symbols above the bars indicate statistically significant differences $(p<0.05)$ when compared with time point controls: ${ }^{*} p<0.05$ versus $0 \mathrm{~h}$ (WT control); ${ }^{*} p<0.05$ versus WT + LPS (time points).

(For figure see next page.) 
Kidney

Blood Pressure

Research

\begin{tabular}{l|l}
\hline Kidney Blood Press Res 2019;44:465-478 \\
\hline DOI: 10.1159/000499685 & $\begin{array}{l}\text { @ 2019 The Author(s). Published by S. Karger AG, Basel } \\
\text { www.karger.com/kbr }\end{array}$ \\
\hline
\end{tabular}

Ye et al.: Caspase-11 and Septic Acute Kidney Injury

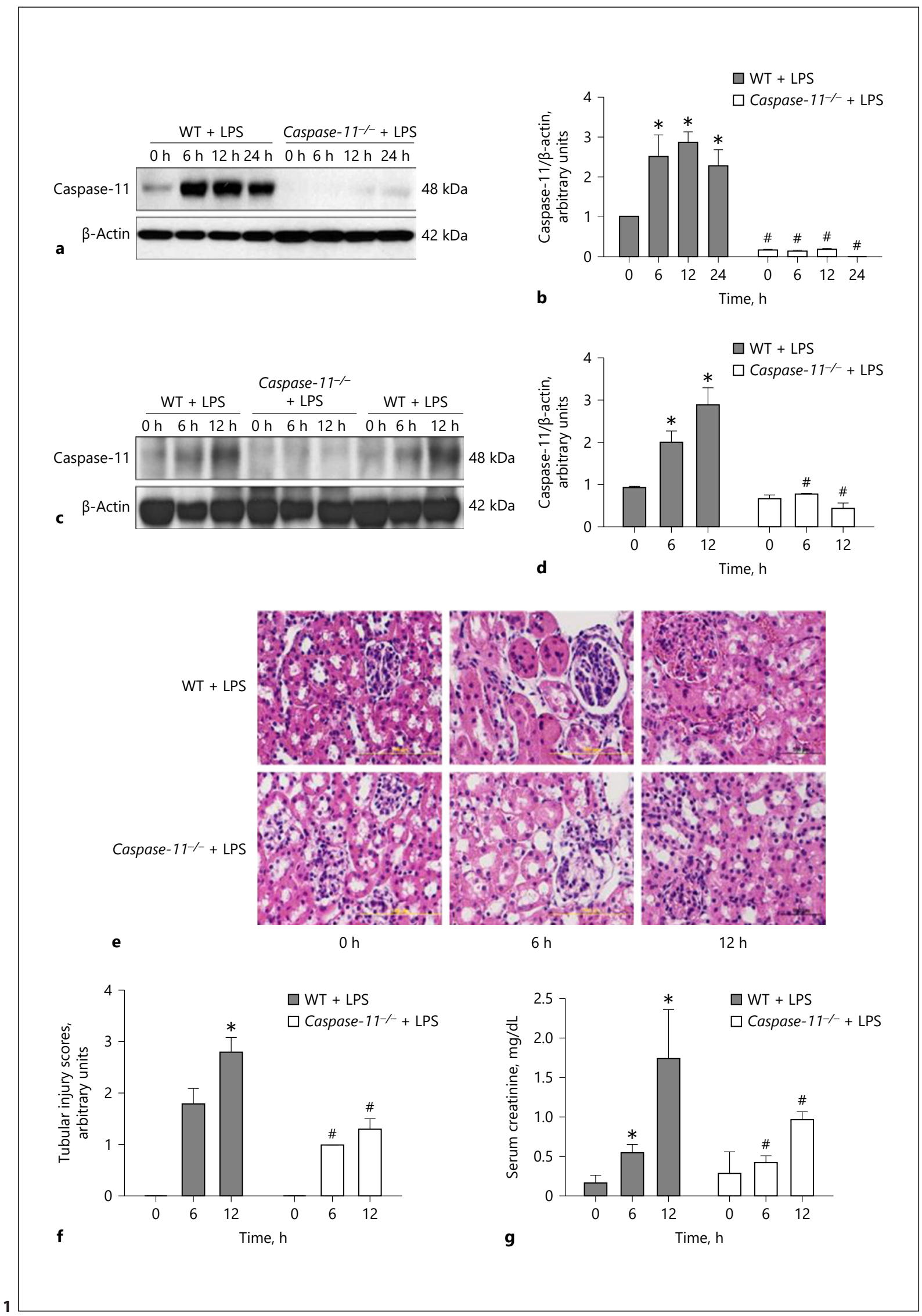




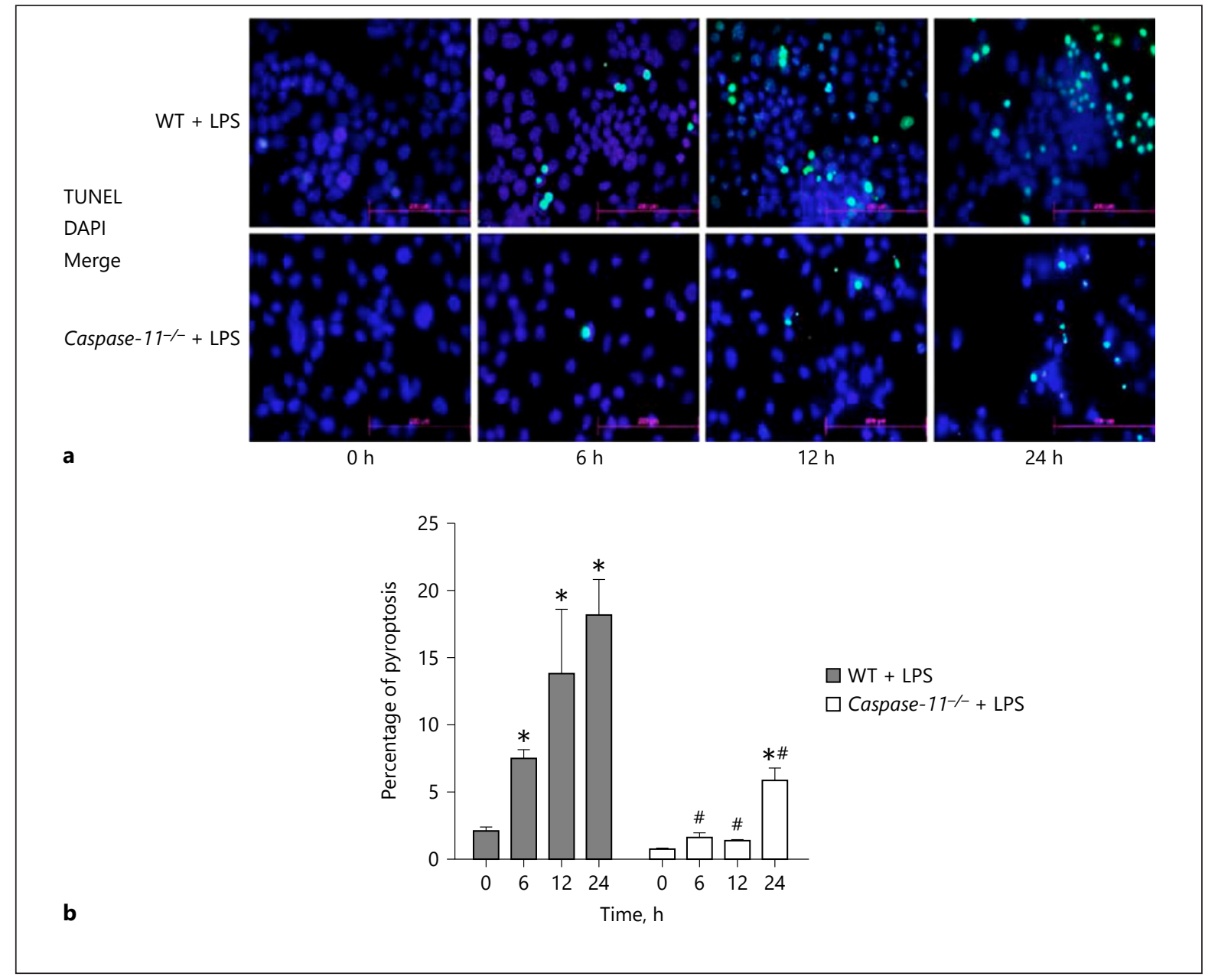

Fig. 2. Pyroptotic cell death of LPS-treated RTECs isolated from wild-type (WT) mice and caspase-11-/- mice. Isolated RTECs from WT mice and caspase-11//- mice were each cultured in vitro and treated with LPS (30 $\mu \mathrm{g} / \mathrm{mL}$ ) for 6, 12, or $24 \mathrm{~h}$. a Pyroptotic cell death was evaluated by TUNEL and 4'-6-diamidino-2-phenylindole (DAPI) staining. b Quantification of three repetitions of pyroptotic cell death in RTECs shown in a. c Pyroptotic cell death was evaluated with flow cytometry. $\mathbf{d}$ Quantification of three repetitions of pyroptotic cell death in RTECs shown in c. Data are expressed as mean \pm SEM. Bars $=200 \mu \mathrm{m}$. ${ }^{*} p<0.05$ versus $0 \mathrm{~h}$ (WT control); ${ }^{*} p<0.05$ versus WT + LPS (time points).

(Figure continued on next page.)

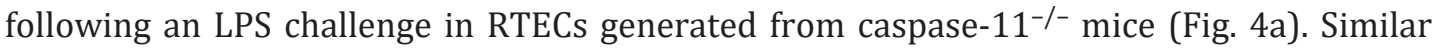
results were observed in in vivo renal tissues following an LPS challenge (Fig. 4b), thus establishing a causal role for caspase-11 as an activator of Gsdmd during AKI. Together, these data show that caspase- 11 is a central mediator for the generation of mature IL- $1 \beta$ and Gsdmd in endotoxic AKI.

Caspase-11 Activation Is Required for Generation of Mature IL-1ß and Gsdmd in Endotoxic Acute Kidney Injury

We next investigated the mechanisms by which caspase-11 activation induces AKI. IL-1 $\beta$ is a pro-inflammatory cytokine which is initially synthesized as a 31-kDa precursor protein and then becomes proteolytically activated by inflammatory caspases. Mature IL- $1 \beta$ protein is released during pyroptotic cell death, thus amplifying the inflammatory cascade $[11,15,19$, 

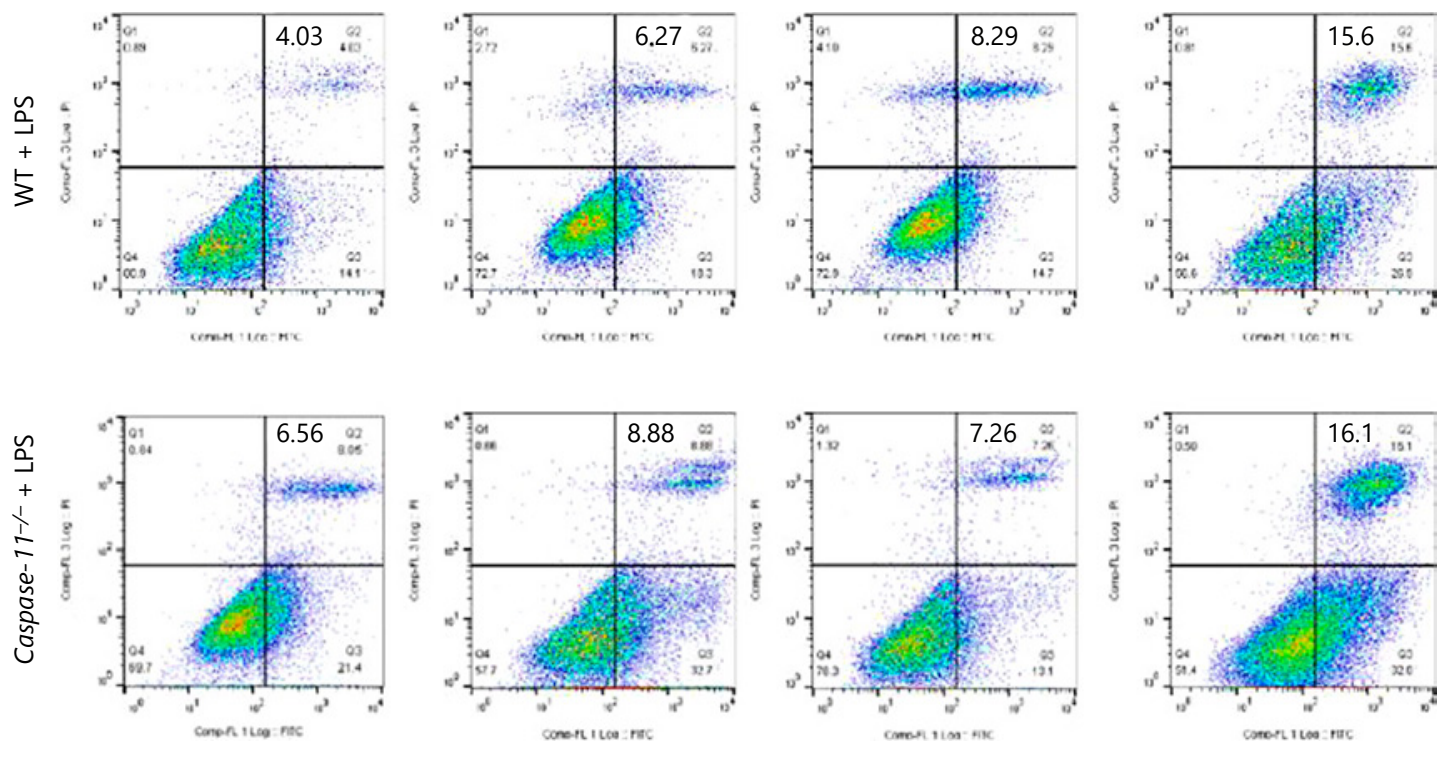

$\mathrm{Oh}$

$6 \mathrm{~h}$

$12 \mathrm{~h}$

$24 \mathrm{~h}$

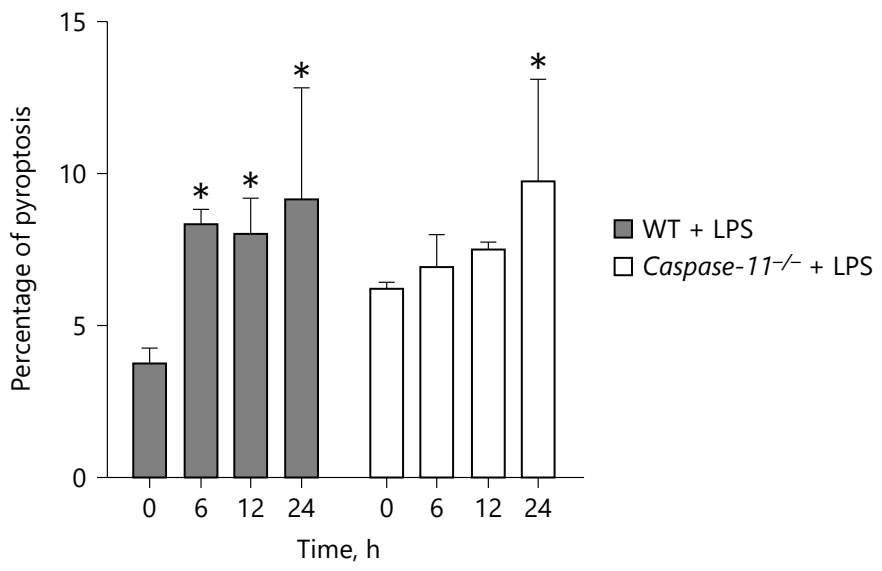

$26,27]$. To determine whether IL- $1 \beta$ is processed by caspase-11 in the kidney, WT and caspase-11//- mice were challenged with LPS (40 mg/kg i.p.) for 6, 12, or $24 \mathrm{~h}$. Kidneys were isolated and IL-1 $\beta$ protein was measured by ELISA. Knockout of caspase-11 resulted in decreased expression of mature IL-1 $\beta$ in kidney tissue following endotoxemia (Fig. 5a). Simi-

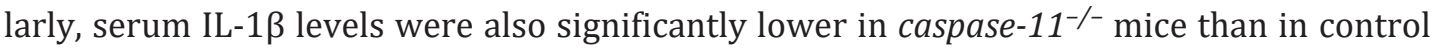
mice after an LPS challenge (Fig. 5b).

Knockout of Caspase-11 Improves Animal Survival after Lipopolysaccharide Stimulation

In WT mice challenged with a lethal dose of LPS ( $40 \mathrm{mg} / \mathrm{kg}$ i.p.), the earliest death event occurred at $20 \mathrm{~h}$ and no animals survived after $28 \mathrm{~h}$. However, caspase-11 knockout mice challenged with a lethal LPS dose experienced no death events by the end of $72 \mathrm{~h}$, and displayed a survival of $70-80 \%$ after 7 days, as demonstrated with Kaplan-Meier survival analysis (Fig. 6). These results demonstrate that knockout of caspase-11 improves animal survival in endotoxemia. 


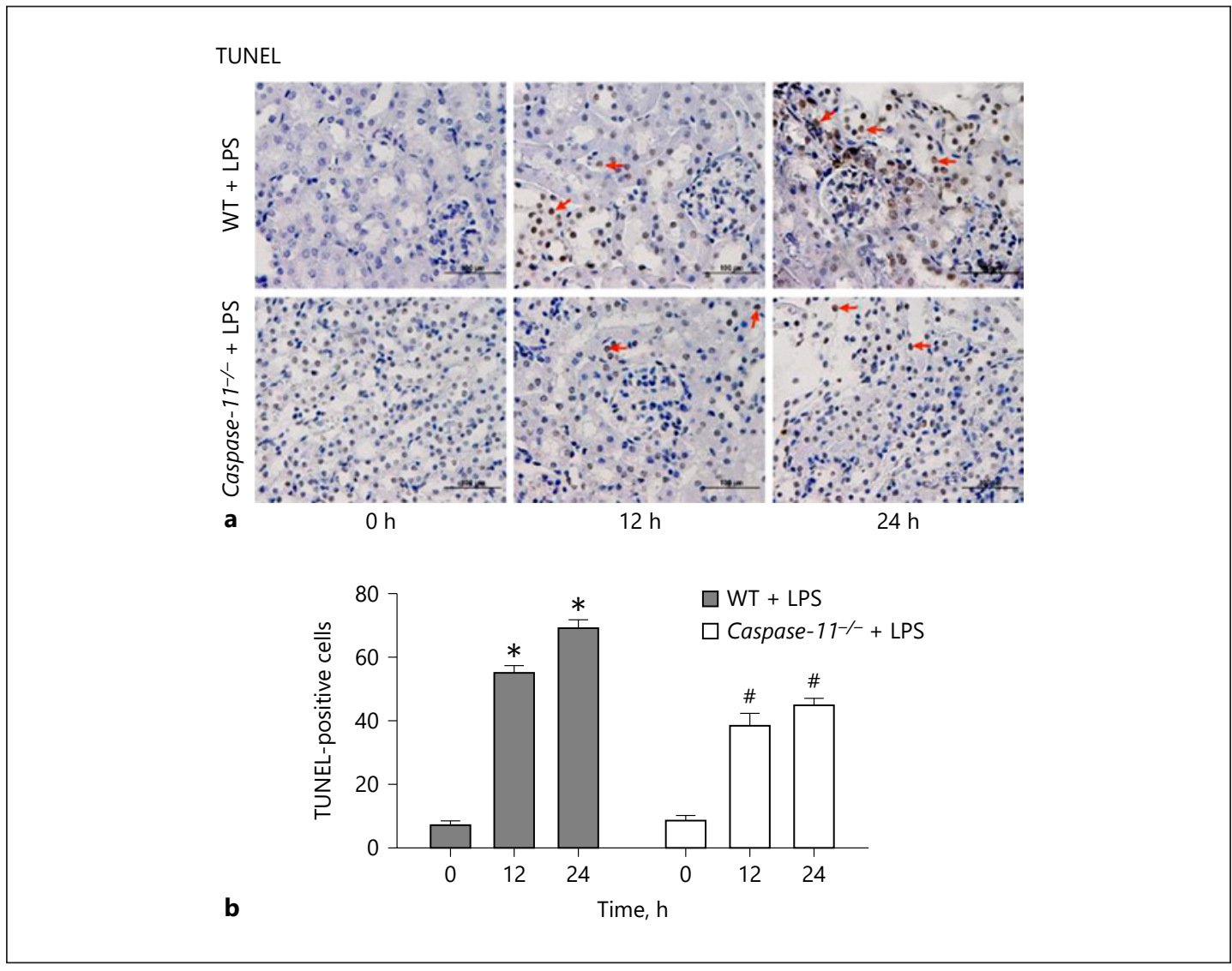

Fig. 3. Pyroptotic cell death of tubular epithelial cells in renal tissue of wild-type (WT) mice and caspase-11/mice after an LPS challenge. Cell death was evaluated by TUNEL staining, and TUNEL-positive cells were counted. Data are expressed as mean \pm SEM, $n=6$. Bars $=100 \mu \mathrm{m} .{ }^{*} p<0.05$ versus $0 \mathrm{~h}$ (WT control); ${ }^{\#} p<$ 0.05 versus WT + LPS (time points).

\section{Discussion}

Sepsis has been identified as the most common cause of AKI in intensive care units. Moreover, the combination of sepsis and AKI is associated with a very high mortality rate [1, 28]. Therefore, there is an urgent need to identify novel therapeutic interventions to attenuate septic AKI. Previous studies [4, 29-32] have shown that apoptosis and necrosis are the two major cell death pathways that contribute to death of RTECs in septic AKI. Pyroptosis is a recently identified pathway of host cell death that is stimulated by a range of microbial infections and non-infectious stimuli [33-35]. However, whether pyroptosis occurs in septic AKI had not been addressed. In the present study, we demonstrated that the endotoxin LPS induces pyroptosis of RTECs. Moreover, LPS treatment induced epithelial caspase-11 activation, which resulted in cleavage of Gsdmd, and ultimately triggered pyroptotic cell death of RTECs. Importantly, these data demonstrate that caspase-11-mediated epithelial pyroptosis is an important pathogenic factor in the development of LPS-induced AKI.

LPS, an endotoxin, is a major component of the outer membrane of gram-negative bacteria, which are considered to be the primary etiology of sepsis. LPS is an ideal model for experimental endotoxemia-induced kidney injury and is widely used to study the mechanisms of septic AKI $[36,37]$. Here, we successfully established a murine AKI model by treating 


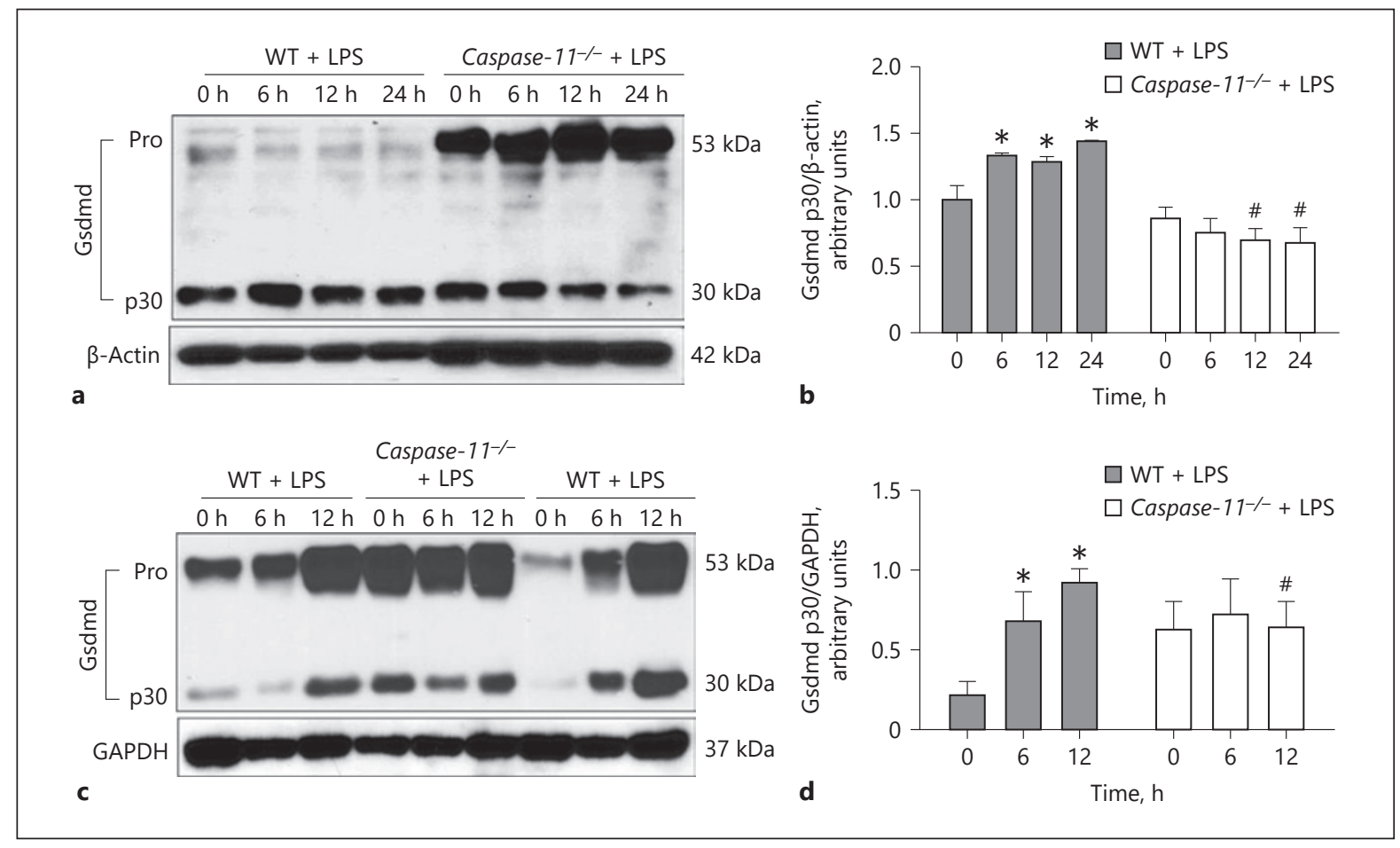

Fig. 4. Cleaved Gsdmd p30 protein expression in RTECs cultured in vitro and in renal tissues. Isolated RTECs

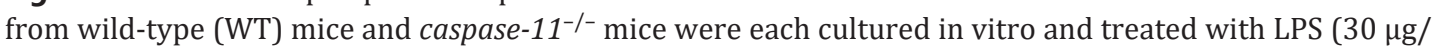

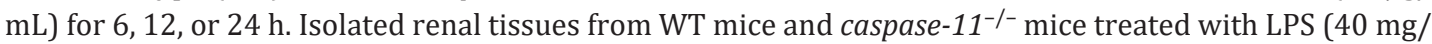
$\mathrm{kg}$ ). a The protein level of cleaved Gsdmd p30 protein was analyzed in RTECs by western blot. b Densitometric analysis of three repetitions of caspase-11 protein in RTECs shown in a. c The protein level of cleaved Gsdmd p30 protein was analyzed in renal tissues by western blot. $\mathbf{d}$ Densitometric analysis of three repetitions of caspase-11 protein in renal tissues shown in c. Different symbols above the bars indicate statistically significant differences $(p<0.05)$ when compared with time point controls: ${ }^{*} p<0.05$ versus $0 \mathrm{~h}$ (WT control); ${ }^{\#} p<0.05$ versus WT + LPS (time points).

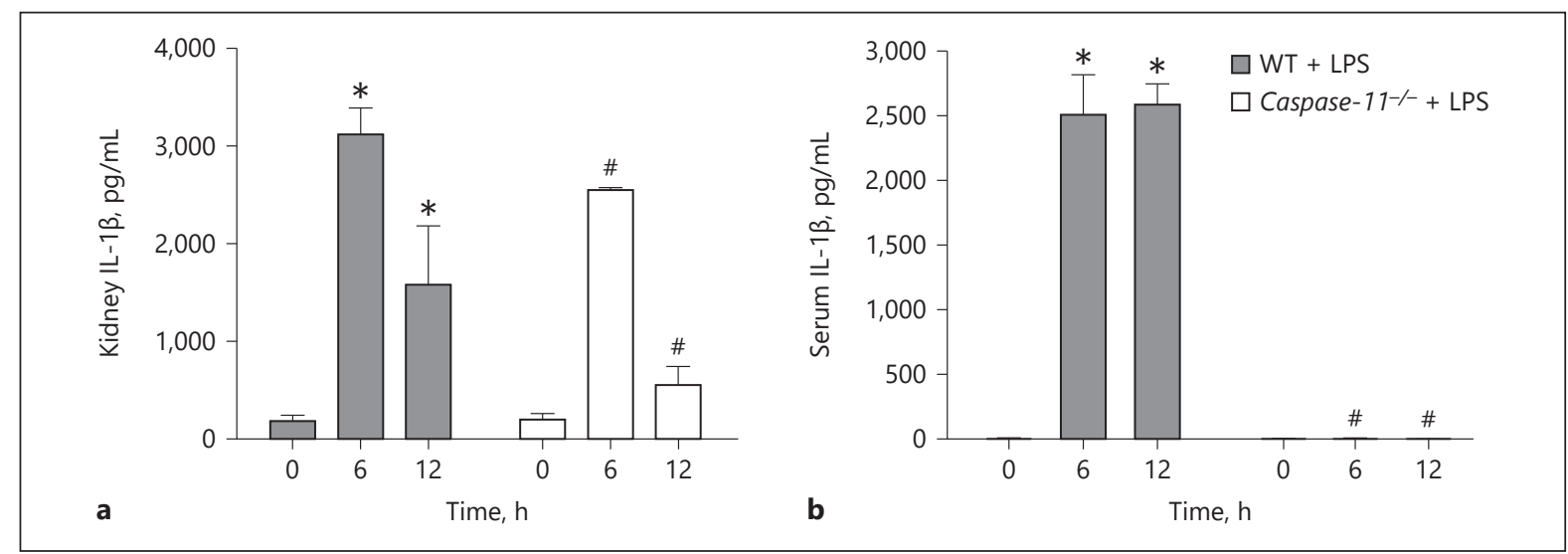

Fig. 5. IL-1 $\beta$ was measured in kidney tissue and serum of wild-type (WT) and caspase-11-/- mice after an LPS challenge. a ELISA analysis of IL-1 $\beta$ in kidney tissue of WT and caspase-11 ${ }^{-/-}$mice treated with LPS for 0,6 , or $12 \mathrm{~h}$. Deletion of caspase-11 decreased IL-1 $\beta$ in kidney tissue following endotoxemia. b IL-1 $\beta$ was mea-

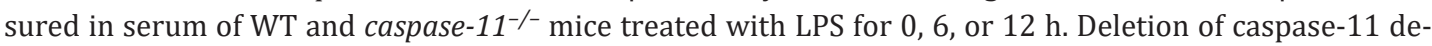
creased serum IL-1 $\beta$ in mice following endotoxemia. Data are expressed as mean \pm SEM, $n=8 . * p<0.05$ versus $0 \mathrm{~h}$ (WT control); ${ }^{*} p<0.05$ versus WT mice + LPS (time points). 
Fig. 6. Kaplan-Meier analysis animal survival after LPS stimulation. Knockout caspase-11 improves animal survival in endotoxemia. ${ }^{*} p<0.05$ versus $0 \mathrm{~h}$ (WT control); ${ }^{\#} p<0.05$ versus WT mice + LPS (time points).

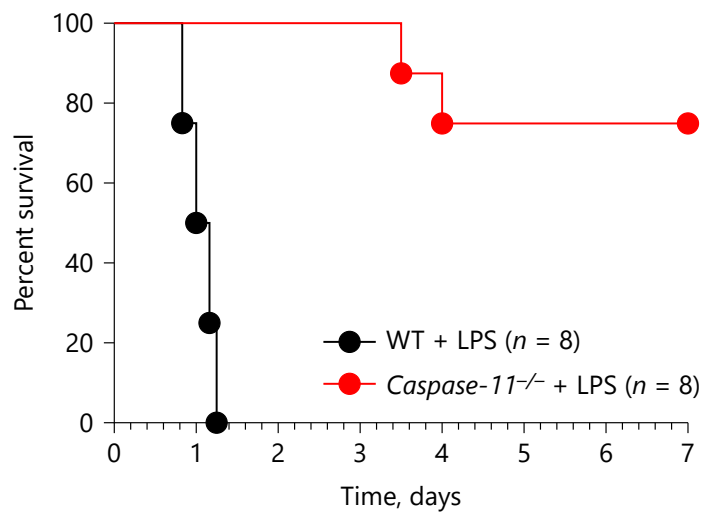

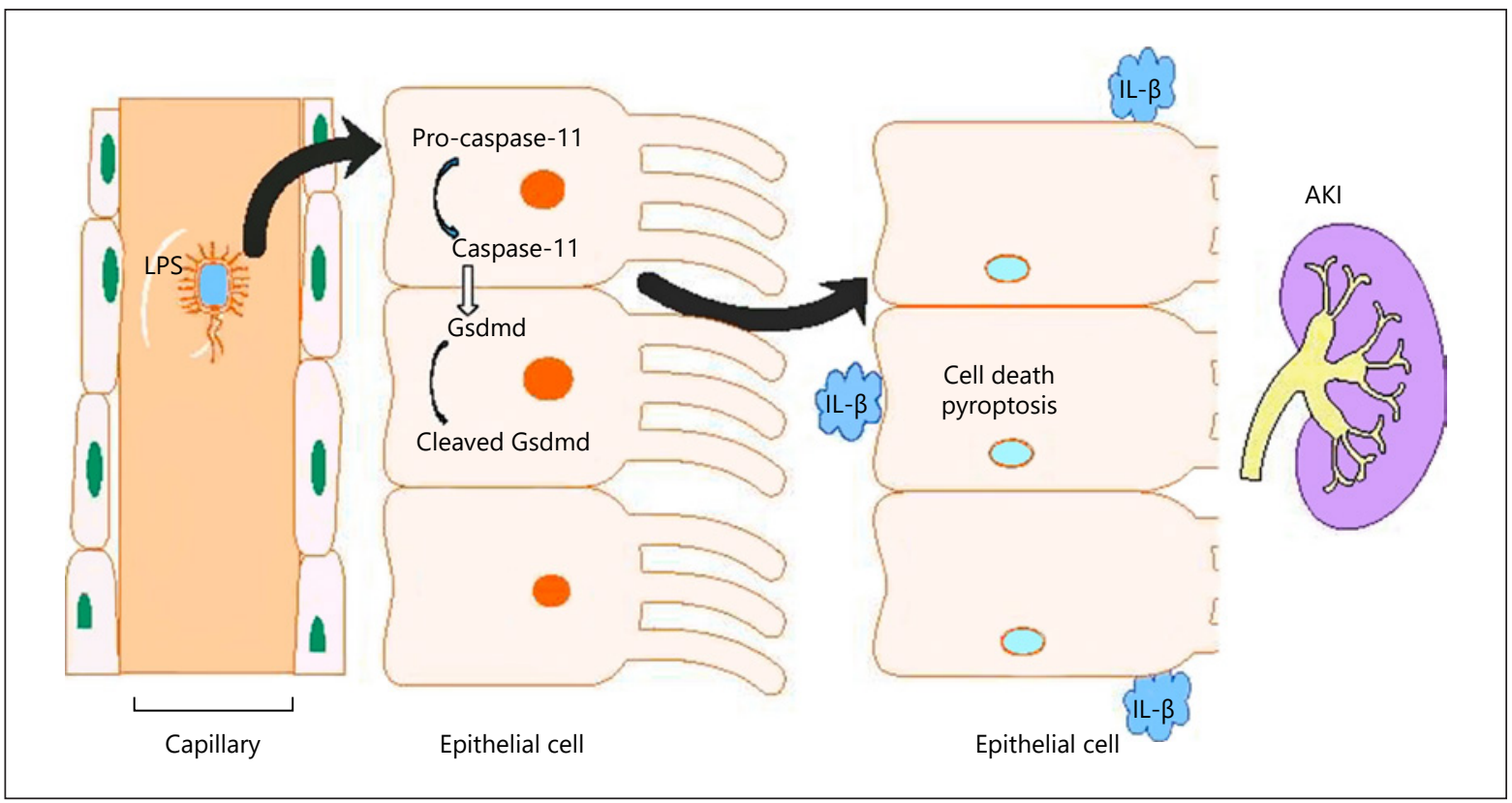

Fig. 7. The proposed mechanism of endotoxin induces AKI. Caspase-11/cleaved Gsdmd mediates endotoxininduced pyroptosis in RTECs.

C57BLk/6J mice with $40 \mathrm{mg} / \mathrm{kg}$ LPS (from Escherichia coli 0111:B4). Histopathological examination showed that, following an LPS challenge in mice, the renal tubular structure was destroyed, RTECs were degenerated, and there was severe intracellular edema within renal tubules as well as in the renal interstitium. In addition, Scr levels, which are used as an index of renal injury, were found to be significantly increased in WT mice after LPS injection. Together, these findings establish that LPS injection is a suitable animal model for AKI.

Pyroptosis plays an important role in the antibacterial innate immune defense and in lethal endotoxemia $[16,38]$. Caspase-11 defines the non-canonical inflammasome that is activated by various gram-negative bacterial infections and causes infected cells to die by pyroptosis [15-19]. A previous study has shown that caspase-11 mediates ischemia-reperfusioninduced renal tubule pyroptosis [39]. In the present study, our findings indicate that caspase-11 is increased in RTECs isolated from endotoxemic WT mice. In addition, we also 


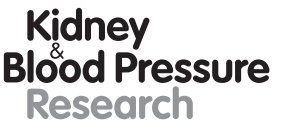

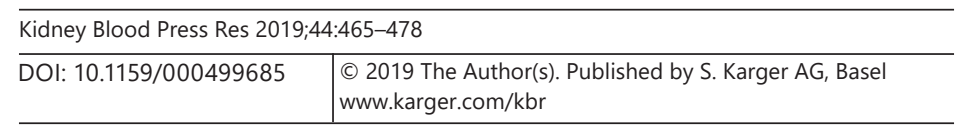

Ye et al.: Caspase-11 and Septic Acute Kidney Injury

observed pyroptotic cell death in LPS-induced AKI. Importantly, endotoxin-induced pyroptosis was alleviated in RTECs after caspase-11 knockout, and Scr was also decreased. Meanwhile, the pathologic aberrance and death events observed following an LPS challenge in WT

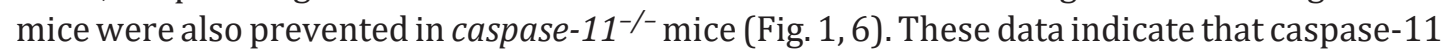
mediates endotoxin-induced renal tubule pyroptosis and kidney injury.

Gsdmd has been recently identified through genetic screens as a key mediator of pore formation in cells undergoing pyroptosis, with pore formation resulting in rapid lytic cell death $[12,15,19,23-25,27]$. Gsdmd is a critical target of caspase-11; its cleavage as well as the resultant $\mathrm{N}$-terminal fragments were shown to be the executioner of pyroptosis $[15,19]$. Here we observed cleavage of the Gsdmd p30 fragment in renal epithelial cells in response to LPS treatment. In addition, we observed a marked increase in the active, cleaved Gsdmd p30 protein in LPS-treated RTECs isolated from WT mice. In contrast, the cleaved Gsdmd p30 protein was decreased in LPS-treated RTECs isolated from caspase-11//- mice, suggesting that epithelial cells undergo pyroptotic cell death via Gsdmd-mediated pore formation.

IL-1 $\beta$, a pro-inflammatory cytokine, is an important mediator in the progression of sepsis. Previous studies identified Gsdmd as a component of the inflammasome responsible for the execution of pyroptosis as well as the secretion of matured IL-1 $\beta$ [25]. In the present study, we show that the endotoxin LPS increased IL-1 $\beta$ in plasma and kidney tissues from WT mice, thus inducing an inflammatory response that results in AKI. However, the serum and kidney IL-1 $\beta$ levels remained low in caspase $-11^{-/-}$mice after an LPS challenge (Fig. 5), suggesting that IL-1 $\beta$ is involved in the progression of septic AKI.

In summary, the present study demonstrates that renal epithelial cells undergo pyroptosis in LPS-induced AKI. Furthermore, we show that inflammatory caspase-11 mediates LPSinduced Gsdmd activation and consequent IL-1 $\beta$ release in RTECs undergoing pyroptosis (Fig. 7). We also show that knockout of caspase-11 preserves renal function, ameliorates pathological injury, and improves mouse survival in LPS-induced AKI. Targeting caspase11-mediated pyroptosis may serve as a new effective therapeutic strategy to attenuate septic AKI.

\section{Acknowledgements}

This study was supported by the grants from the Natural Science Foundation of Guangdong Province (2018A0303130336) and National Natural Science Foundation of China (Grants 81600575; 81570609; 81470930; 81400738).

\section{Statement of Ethics}

All animals were treated in accordance with the National Institutes of Health guidelines for use and care of research animals.

\section{Disclosure Statement}

No potential conflicts of interest relevant to this article were reported. 


\begin{tabular}{l|l}
\hline \multicolumn{2}{l}{ Kidney Blood Press Res 2019;44:465-478 } \\
\hline DOI: 10.1159/000499685 & $\begin{array}{l}\text { @ 2019 The Author(s). Published by S. Karger AG, Basel } \\
\text { www.karger.com/kbr }\end{array}$ \\
\hline
\end{tabular}

Ye et al.: Caspase-11 and Septic Acute Kidney Injury

\section{References}

1 Uchino S, Kellum JA, Bellomo R, Doig GS, Morimatsu H, Morgera S, et al.; Beginning and Ending Supportive Therapy for the Kidney (BEST Kidney) Investigators. Acute renal failure in critically ill patients: a multinational, multicenter study. JAMA. 2005 Aug;294(7):813-8.

2 Bellomo R, Kellum JA, Ronco C, Wald R, Martensson J, Maiden M, et al. Acute kidney injury in sepsis. Intensive Care Med. 2017 Jun;43(6):816-28.

3 Kaddourah A, Basu RK, Bagshaw SM, Goldstein SL; AWARE Investigators. Epidemiology of Acute Kidney Injury in Critically Ill Children and Young Adults. N Engl J Med. 2017 Jan;376(1):11-20.

4 Havasi A, Borkan SC. Apoptosis and acute kidney injury. Kidney Int. 2011 Jul;80(1):29-40.

5 Linkermann A, Chen G, Dong G, Kunzendorf U, Krautwald S, Dong Z. Regulated cell death in AKI. J Am Soc Nephrol. 2014 Dec;25(12):2689-701.

6 Linkermann A. Nonapoptotic cell death in acute kidney injury and transplantation. Kidney Int. 2016 Jan;89(1): 46-57.

7 Lamkanfi M, Dixit VM. Inflammasomes and their roles in health and disease. Annu Rev Cell Dev Biol. 2012; 28(1):137-61.

8 Fink SL, Cookson BT. Apoptosis, pyroptosis, and necrosis: mechanistic description of dead and dying eukaryotic cells. Infect Immun. 2005 Apr; 73(4):1907-16.

9 Miao EA, Rajan JV, Aderem A. Caspase-1-induced pyroptotic cell death. Immunol Rev. 2011 Sep;243(1):20614.

10 Jorgensen I, Rayamajhi M, Miao EA. Programmed cell death as a defence against infection. Nat Rev Immunol. 2017 Mar;17(3):151-64.

11 Yuan J, Najafov A, Py BF. Roles of Caspases in Necrotic Cell Death. Cell. 2016 Dec;167(7):1693-704.

12 He WT, Wan H, Hu L, Chen P, Wang X, Huang Z, et al. Gasdermin D is an executor of pyroptosis and required for interleukin-1 $\beta$ secretion. Cell Res. 2015 Dec;25(12):1285-98.

13 Shi J, Zhao Y, Wang Y, Gao W, Ding J, Li P, et al. Inflammatory caspases are innate immune receptors for intracellular LPS. Nature. 2014 Oct;514(7521):187-92.

14 Cheng KT, Xiong S, Ye Z, Hong Z, Di A, Tsang KM, et al. Caspase-11-mediated endothelial pyroptosis underlies endotoxemia-induced lung injury. J Clin Invest. 2017 Nov;127(11):4124-35.

15 Kayagaki N, Stowe IB, Lee BL, O’Rourke K, Anderson K, Warming S, et al. Caspase-11 cleaves gasdermin D for non-canonical inflammasome signalling. Nature. 2015 Oct;526(7575):666-71.

16 Kayagaki N, Warming S, Lamkanfi M, Vande Walle L, Louie S, Dong J, et al. Non-canonical inflammasome activation targets caspase-11. Nature. 2011 Oct;479(7371):117-21.

17 Kayagaki N, Wong MT, Stowe IB, Ramani SR, Gonzalez LC, Akashi-Takamura S, et al. Noncanonical inflammasome activation by intracellular LPS independent of TLR4. Science. 2013 Sep;341(6151):1246-9.

18 Hagar JA, Powell DA, Aachoui Y, Ernst RK, Miao EA. Cytoplasmic LPS activates caspase-11: implications in TLR4-independent endotoxic shock. Science. 2013 Sep;341(6151):1250-3.

19 Shi J, Zhao Y, Wang K, Shi X, Wang Y, Huang H, et al. Cleavage of GSDMD by inflammatory caspases determines pyroptotic cell death. Nature. 2015 Oct;526(7575):660-5.

20 Broz P. Immunology: caspase target drives pyroptosis. Nature. 2015 Oct;526(7575):642-3.

21 Aachoui Y, Leaf IA, Hagar JA, Fontana MF, Campos CG, Zak DE, et al. Caspase-11 protects against bacteria that escape the vacuole. Science. 2013 Feb;339(6122):975-8.

22 Paller MS, Hoidal JR, Ferris TF. Oxygen free radicals in ischemic acute renal failure in the rat. J Clin Invest. 1984 Oct; 74(4):1156-64.

23 Ding J, Wang K, Liu W, She Y, Sun Q, Shi J, et al. Pore-forming activity and structural autoinhibition of the gasdermin family. Nature. 2016 Jul;535(7610):111-6.

24 Sborgi L, Rühl S, Mulvihill E, Pipercevic J, Heilig R, Stahlberg H, et al. GSDMD membrane pore formation constitutes the mechanism of pyroptotic cell death. EMBO J. 2016 Aug;35(16):1766-78.

25 Liu X, Zhang Z, Ruan J, Pan Y, Magupalli VG, Wu H, et al. Inflammasome-activated gasdermin D causes pyroptosis by forming membrane pores. Nature. 2016 Jul;535(7610):153-8.

26 Jorgensen I, Miao EA. Pyroptotic cell death defends against intracellular pathogens. Immunol Rev. 2015 May; 265(1):130-42.

27 Aglietti RA, Estevez A, Gupta A, Ramirez MG, Liu PS, Kayagaki N, et al. GsdmD p30 elicited by caspase-11 during pyroptosis forms pores in membranes. Proc Natl Acad Sci USA. 2016 Jul;113(28):7858-63.

28 Schrier RW, Wang W. Acute renal failure and sepsis. N Engl J Med. 2004 Jul;351(2):159-69.

29 Gómez H, Kellum JA. Sepsis-induced acute kidney injury. Curr Opin Crit Care. 2016 Dec;22(6):546-53.

30 Luo CJ, Luo F, Zhang L, Xu Y, Cai GY, Fu B, et al. Knockout of interleukin-17A protects against sepsis-associated acute kidney injury. Ann Intensive Care. 2016 Dec;6(1):56.

31 Pickkers P, Ostermann M, Joannidis M, Zarbock A, Hoste E, Bellomo R, et al. The intensive care medicine agenda on acute kidney injury. Intensive Care Med. 2017 Sep;43(9):1198-209.

32 Holthoff JH, Wang Z, Seely KA, Gokden N, Mayeux PR. Resveratrol improves renal microcirculation, protects the tubular epithelium, and prolongs survival in a mouse model of sepsis-induced acute kidney injury. Kidney Int. 2012 Feb;81(4):370-8.

33 Bergsbaken T, Fink SL, Cookson BT. Pyroptosis: host cell death and inflammation. Nat Rev Microbiol. 2009 Feb;7(2):99-109. 
34 Jorgensen I, Rayamajhi M, Miao EA. Programmed cell death as a defence against infection. Nat Rev Immunol. 2017 Mar; 17(3):151-64.

35 Wang Y, Gao W, Shi X, Ding J, Liu W, He H, et al. Chemotherapy drugs induce pyroptosis through caspase-3 cleavage of a gasdermin. Nature. 2017 Jul;547(7661):99-103.

36 Doi K, Leelahavanichkul A, Yuen PS, Star RA. Animal models of sepsis and sepsis-induced kidney injury. J Clin Invest. 2009 Oct; 119(10):2868-78.

37 Schultz MJ, van der Poll T. Animal and human models for sepsis. Ann Med. 2002;34(7-8):573-81.

38 Miao EA, Leaf IA, Treuting PM, Mao DP, Dors M, Sarkar A, et al. Caspase-1-induced pyroptosis is an innate immune effector mechanism against intracellular bacteria. Nat Immunol. 2010 Dec;11(12):1136-42.

39 Yang JR, Yao FH, Zhang JG, Ji ZY, Li KL, Zhan J, et al. Ischemia-reperfusion induces renal tubule pyroptosis via the CHOP-caspase-11 pathway. Am J Physiol Renal Physiol. 2014 Jan;306(1):F75-84. 